\title{
Tracking and synchronization of the yeast cell cycle using dielectrophoretic opacity $\dagger+$
}

\author{
Ana Valero, $\oint^{* a}$ Thomas Braschler, $\oint^{* a}$ Alex Rauch, ${ }^{b}$ Nicolas Demierre, ${ }^{a}$ Yves Barral $^{b}$ and Philippe Renaud ${ }^{a}$ \\ Received 6th January 2011, Accepted 10th March 2011 \\ DOI: 10.1039/c1lc00007a \\ Cell cycle synchronization is an important tool for the study of the cell division stages and signalling. It \\ provides homogeneous cell cultures that are of importance to develop and improve processes such as \\ protein synthesis and drug screening. The main approach today is the use of metabolic agents that \\ block the cell cycle at a particular phase and accumulate cells at this phase, disturbing the cell \\ physiology. We provide here a non-invasive and label-free continuous cell sorting technique to analyze \\ and synchronize yeast cell division. By balancing opposing dielectrophoretic forces at multiple \\ frequencies, we maximize sensitivity to the characteristic shape and internal structure changes \\ occurring during the yeast cell cycle, allowing us to synchronize the culture in late anaphase.
}

The ability to reproduce is a fundamental property of life. Mitosis as the means of cellular reproduction of eukaryotic cells has been the subject of intense investigations. A large part of this research has been done using budding yeast cells because of their well-known genetics, robustness and characteristic shape changes during the mitotic cell cycle, ${ }^{1-3}$ allowing the development of a broad range of large-scale methods to study eukaryotic cell biochemistry. ${ }^{4}$ Many of these approaches depend on cell synchronization in order to dissect mechanisms specific to certain stages of the cell cycle. For yeast cells, two major types of synchronization methods are known:, 5 invasive methods synchronize whole cultures by means of cell cycle arrest induced by mating factor alpha ${ }^{7}$ or a temperature rise in conditional mutants, ${ }^{8}$ whereas elutriation selects a subset of small, and hence young cells. ${ }^{9,10}$ Cell cycle arrest methods yield highly synchronized cultures, but as detailed gene expression analysis shows, they also interfere with the natural cell cycle, leading to cyclic expression patterns that might not exist in freely growing yeast. ${ }^{10}$ For detailed investigation of the unperturbed cell division machinery, it would be desirable to dispose of a non-invasive selection method providing cells in active division in addition to the young cells accessible by elutriation. We demonstrate the synchronization of a yeast cell culture in late anaphase by using electrical forces based on dielectrophoresis. The technique makes

${ }^{a}$ Microsystems Laboratory, Batiment de Microtechnique Station 17, Swiss Federal Institute of Technology, CH-1015 Lausanne, Switzerland. E-mail: ana.valero@epfl.ch; thomas.braschler@epfl.ch

${ }^{b}$ Institute of Biochemistry, Schafmattstr. 18, Swiss Federal Institute of Technology, CH-8093 Zurich, Switzerland

$\uparrow$ Competing interests: the authors declare that they have no competing financial interests.

\$ Electronic supplementary information (ESI) available. See DOI: $10.1039 / \mathrm{c} 1 \mathrm{lc} 00007 \mathrm{a}$

$\S$ These authors contributed equally. use of the characteristic variation of cell geometry and structure, ${ }^{3,11}$ and hence of the electrical properties of budding yeast cells throughout their division cycle, ${ }^{12}$ to achieve continuous sorting on chip.

Dielectrophoresis designates the force exerted on particles by inhomogeneous, and generally alternating, electric fields. ${ }^{13}$ This dielectrophoretic force is due to the action of the inhomogeneous field on the dipole induced on the particle; hence, dielectrophoresis reflects the particles' effective permittivity as compared to the suspension medium. ${ }^{14}$ For cells in suspension, the dielectrophoretic force depends on four main factors: ${ }^{14-16}$ the medium composition, the intrinsic electric parameters of the constituents of the cells, the cell volume and the cell geometry. The first two factors, given by the medium conductivity and permittivity, and the intrinsic electric parameters of the cell wall, cell membrane and cytosol are expected to vary little throughout the cell cycle. Cell volume and geometry on the other hand change in a characteristic manner through out the division cycle due to cell growth and budding. The joint variation of cell volume and geometry makes the task of sorting yeast division stages using dielectrophoresis more challenging than sorting division stages of mammalian cells in suspension, ${ }^{17}$ which can be assumed to retain an approximately spherical shape throughout most of the cycle.

Cell cycle progression in yeast is tightly correlated to bud size ${ }^{3}$ suggesting that a strategy based on cell geometry rather than cell volume should yield efficient dielectrophoretic sorting of yeast division stages. We use an equilibrium between dielectrophoretic forces produced by electric fields of different frequencies ${ }^{18}$ acting from both sides of a sorting channel, where the suspended yeast cells are flowing (Fig. 1-A). This opposition of dielectrophoretic forces eliminates the first order dependence of the force on the volume, ${ }^{19}$ increasing the sensitivity to changes in cell shape throughout the division cycle. The arrangement has two additional important advantages: ${ }^{18,20}$ First, the cell sorting takes 


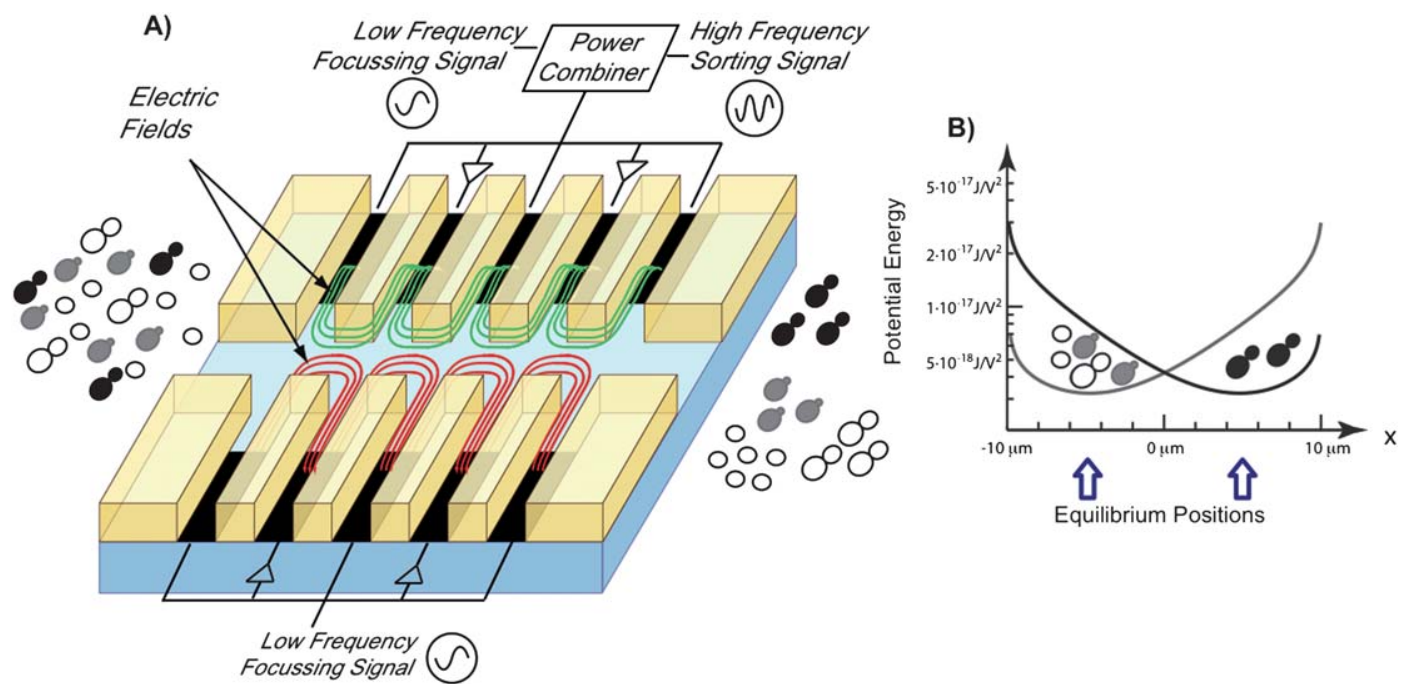

Fig. 1 On chip continuous cell sorting based on equilibrium dielectrophoresis. (A) Scheme of the sorting unit; a combination of platinum electrodes (black rectangles) with a photopatterned insulator (yellow blocks) is used to generate an electric field (green and red curved lines) within the central channel where cells flow. Two opposing DEP-forces are created by applying single- or multiple-frequencies signals to the electrodes. Cells get sorted based on their specific dielectric properties at different stages of the cell cycle. (B) Equilibrium-based DEP sorting; for a given set of electric fields the forces acting on the cells from either side of the channel are specific for the cell cycle stage. The ratio of these forces describes the relative efficiency of either electric field to push the cells towards the opposite side of the channel. Sorting of a non-synchronized cell population is achieved as shown in Fig. 1A; non-dividing yeasts $(\bigcirc)$, early stage division yeast $\left(\bigcirc^{\circ}\right)$ and yeast doublets having already undergone cell separation by cytokinesis $\left({ }^{\rho}\right)$ are focused towards a position close to the left channel wall, whereas the minimum of the potential well for the late metaphase and anaphase divsion stages ( $)$ is located towards the right channel wall. The sorting occurs continuously because each division stage is centred towards its own equilibrium position when flowing down the active element.

place perpendicular to the flow, such that the device can be operated continuously; and second, the cells are pushed towards a characteristic equilibrium position for the different division stages, independently of the flow speed (Fig. 1-B). We quantify the dielectric response of the different yeast cell cycle stages as estimated from the dielectrophoretic equilibrium positions in the microfluidic sorting device. Based on this knowledge, we synchronize a yeast cell culture in late anaphase and show that the cell synchrony is maintained during the following divisions. We also provide a detailed theoretical framework describing the dielectrophoretic changes occurring during the yeast cell division cycle, based on the characteristic geometrical changes occurring during the budding process.

\section{Results and discussion}

\section{Dielectrophoretic yeast sorting}

By letting a yeast cell suspension containing different cell division stages flow through the sorting device shown in Fig. 1-A, cells with different morphology or internal structure are sorted to different output positions, as outlined in Fig. 1-B. If there is a difference in the dielectric response for the different cell cycle stages, they should be focused towards different equilibrium position after the sorting element.

A simple indicator of the cell cycle progression in budding yeast is the bud size, which we use to test the applicability of equilibrium-dielectrophoresis for cell cycle synchronization. In G1 yeast cells are spherical and do not have a bud. Bud formation is initiated in S phase, and growth of the bud correlates with cell cycle progression. Finally, the two cells are separated by neck closure due to new membrane and wall synthesis during cytokinesis. Hence, quantifying the relative size of mother and daughter cells allows a rough determination of the cell cycle stage. Fig. 2-A shows the relationship of the bud-to-mother ratio with the dielectrophoretic equilibrium position. The population of cells with medium-sized buds, labelled 2 in the figure, is focused towards the left hand side of the sorting channel, having the most negative $x$ values. The non-dividing cells, and cells with smaller or larger buds (G1-metaphase cells, and cells after cytokinesis), occupy positions closer to the channel midline (see also the movie in the ESIt). This result suggests that indeed, it is possible to use equilibrium dielectrophoresis to synchronize a yeast cell culture.

The spatial positions obtained by microscopic observation are indicative of the dielectric properties of the yeast cells. In the following we provide the theory required to relate these positions, which are dependent on the particular chip geometry and the applied voltages, to the fundamental dielectric properties responsible for the dielectrophoretic cell sorting.

The sorting scheme shown in Fig. 1 implies the use of an equilibrium between dielectrophoretic forces arising from fields at different frequencies rather than a single force at a single frequency. This allows us to avoid the intrinsically strong volume dependency of the dielectric force, and hence to enhance the sensitivity of the output position to the intrinsic dielectric properties of the cell cycle stages. Indeed, other than being dependent on the cell volume $V$, the dielectrophoretic force acting on a cell is also proportional to the Clausius-Mossotti factor $f_{\mathrm{CM}}$, reflecting the intrinsic dielectric properties of the cell: 
A) Sorting as a function of cell geometry

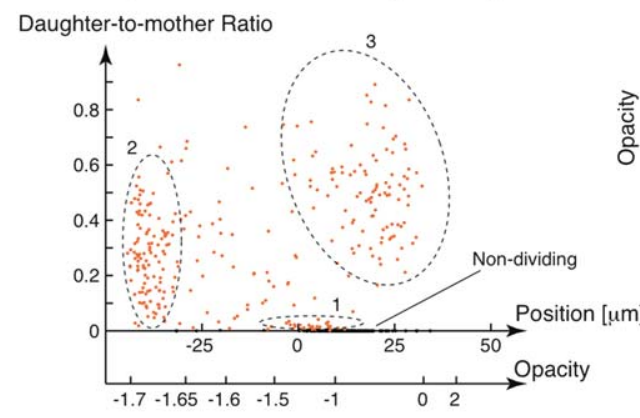

B) Analysis using a GFP-tubulin fusion mutant

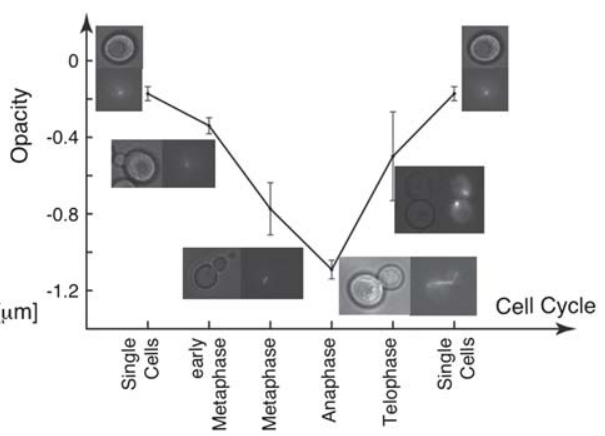

Fig. 2 The dielectric life history of yeast cells. (A) Scatter plot indicating the positions across the outlet channel for non-dividing and dividing yeast cells. Doublets with medium sized buds (2) occupy an extreme position towards the left-hand side of the channel, whereas non-dividing cells, and doublets with smaller (1) and larger buds (3) are directed to the right-hand side of the outlet (positive $x$ values). This is indicative of a relatively stronger pDEP force at high frequency for the doublets with a medium sized bud. (B) Direct relationship between the yeast microtubule cytoskeleton and the variation of the opacity throughout the yeast cell cycle. Spindle length and position are specific for a certain cell cycle stage. Therefore, we can link cell opacity to the different stages of the cell cycle. The graph represents the average and standard deviation of opacity values for all stages of the cell cycle. The generally less negative opacity values in Fig. 2-B are due to the smaller size of the haploid GFP mutant used as compared to the diploid strain employed for Fig. 2-A.

$$
\vec{F}_{\mathrm{DEP}}=\varepsilon_{m} \cdot V \cdot \Re\left(f_{\mathrm{CM}}\right) \cdot \nabla|\vec{E}|^{2}
$$

where $\varepsilon_{m}$ is the permittivity of the suspension medium, $\Re$ designates the real part operator, and $\vec{E}$ is the electric field.

For any particle, the equilibration of two opposing dielectrophoretic forces eliminates the volume dependency and the force ratio depends only on the dielectric opacity of the particle, $\Omega .{ }^{19}$ For equal primary field gradients $\nabla|E|^{2}$ acting at two different frequencies $\omega_{1}$ and $\omega_{2}$, the force ratio is equal to the dielectrophoretic opacity as defined by the ratio of the real parts of the Clausius-Mossotti factor at the respective frequencies:

$$
\frac{F_{\mathrm{DEP}}\left(\omega_{1}\right)}{F_{\mathrm{DEP}}\left(\omega_{2}\right)}=\frac{\Re\left\{f_{\mathrm{CM}}\left(\omega_{1}\right)\right\}}{\Re\left\{f_{\mathrm{CM}}\left(\omega_{2}\right)\right\}}=\Omega\left(\omega_{1}, \omega_{2}\right)
$$

The force ratio on the sorting channel midline determines the lateral equilibrium positions of the particles; vice versa, the force ratio and therefore the yeasts' opacity at a given set of frequencies $\omega_{1}$ and $\omega_{2}$ can be estimated from the experimentally observed output positions of the cells after the sorting element. Experimentally, two factors need to be taken into account for an accurate conversion between opacity and cell output positions: first, we use a channel enlargement for observation of the output positions, resulting in fluidic redistribution due to a change of the channel aspect ratio. Secondly we apply a combined signal on one channel side (as shown in Fig. 1-A) to avoid cells being drawn into the electrode chambers of the sorting device. The details for the conversion between output positions and opacity values are given in Supplementary Information 1.\$ The important point is that for a given chip, and a given set of applied voltages and frequencies, there is a one-to-one relation between the equilibrium position of the particle stream and the opacity value. The opacity values for the signals acting at $100 \mathrm{kHz}$ and 4 $\mathrm{MHz}$ used in Fig. 2-A are given on the secondary $x$-axis. The cell opacities are generally negative. This is due to the transition from repulsive dielectrophoretic forces (nDEP) to attractive DEP forces (pDEP) occurring at about $700 \mathrm{kHz}$ for the medium conductivity of $60 \mathrm{mS} \mathrm{m}^{-1}$, which was used in the entire study.
The largest negative values for opacity are observed for cells with medium sized buds, indicating that these cells experience the relatively strongest pDEP at high frequency. Medium sized buds are observed in late metaphase and anaphase. To assess the influence of the cell cycle stage on the dielectrophoretic opacity value more precisely, we use an additional marker to determine the different cell cycle phases of yeast cells. We use yeast cells expressing GFP-tubulin (see Methods) to visualize microtubules and the spindle. Spindle length and position as well as microtubule length, number and orientation are specific for every phase in the cell cycle. ${ }^{21,22}$ In Fig.2-B mean opacity values are depicted for the different cell cycle stages assessed by fluorescent microscopy (sample size $=40$ ). As cells progress from G1 to anaphase, opacity values become more negative. In telophase opacity values are highly variable, and increase to values measured for G1 cells. This is most likely due to the fact that at this stage cytokinesis takes place, which leads to cell fission and hence electrical insulation of the two cells. Cell division stages explain $r^{2}=66 \%$ of the variance of opacity values. Analysis of variance gives $p=2.6 \times 10^{-8}$, indicating that the division stage is highly correlated with opacity.

\section{Synchronization}

Selecting the fraction of cells with the most negative opacity values yields a synchronized culture of anaphase cells (Fig. 2-B). To assess synchronization quality, we direct a fraction of about $5 \%$ of the cells with the most negative opacity values into an outlet channel, and let them attach to the surface while continuing slow perfusion with YPD yeast cell culture medium. Fig. 3-A shows the fraction of cells collected after the sorting element by sedimentation. The collected cell population contains mostly large budded cells and the error rate in terms of unbudded cells is about $1-2 \%$ for cell populations consisting of $70 \%$ unbudded and 30\% budded cells (see Methods). Fig. 3-B shows time-lapse images of a subpopulation of sorted cells. The cells progress synchronously through following division cycles. They progress from a initial 2-cell stage to 3 cells per colony 
A) Yeast Cell Doublets collected after sorting

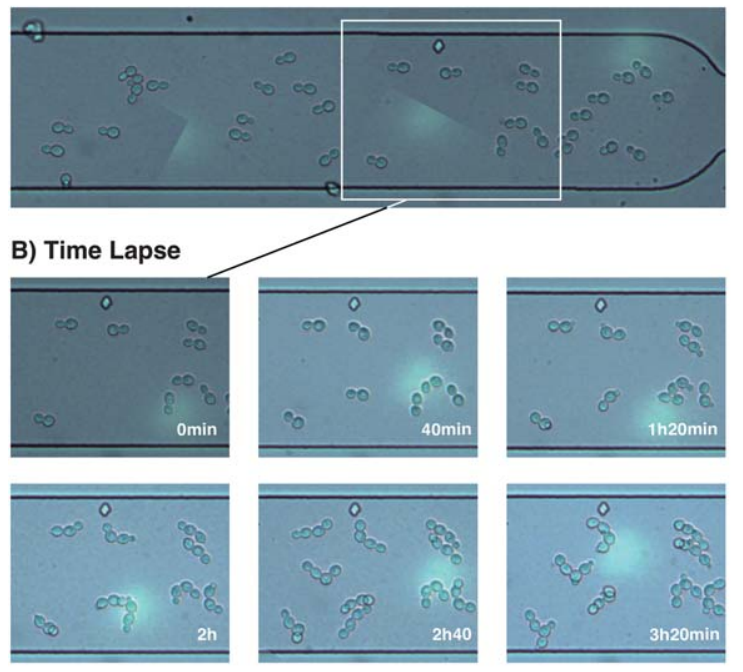

C) Quantification of division activity

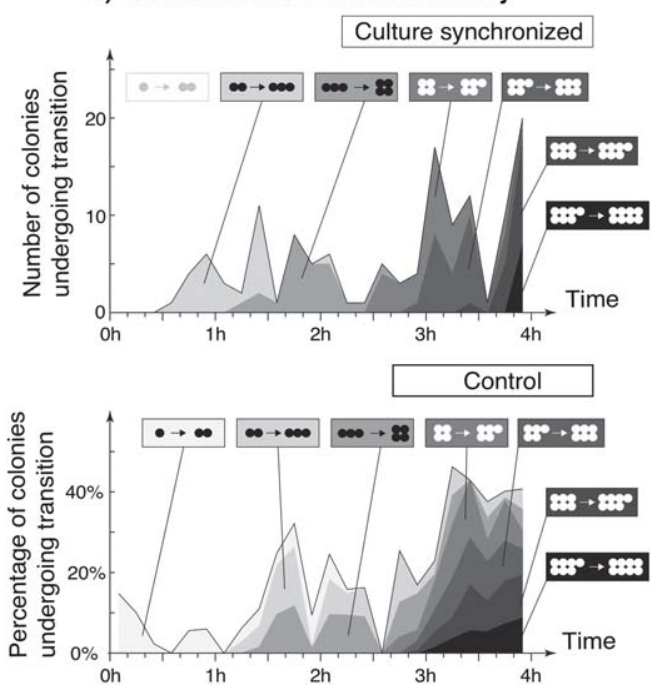

Fig. 3 Synchronization of a yeast cell culture using dielectrophoretic sorting. (A) Yeast cells synchronized at the anaphase state collected at one of the outlet channels of the microfluidic cell-sorting device. (B) Time lapse images, showing cell cycle progression of synchronized cells. (C) Quantification of the cell synchronization in terms of the division activity, quantified as transition to higher cell-number colonies as a function of time.

( $1 \mathrm{~h} 20 \mathrm{~min}$ ), then 4 (initial bud formation at $2 \mathrm{~h}$, all four cells reaching mature size at $2 \mathrm{~h} 40 \mathrm{~min}$ ) and then further to 5 and 6 cell colonies ( 3 h $20 \mathrm{~min}$ ). Fig. 3-C provides a quantitative evaluation of the synchronization, by comparing progression towards higher cell-number colonies for a culture synchronized by dielectrophoresis, and an unsynchronized control culture. In the synchronized cell culture distinct transitions occur from the initial 2-cell stage to the 3- and 4-cell colonies. The transitions to the 5- and 6-cell stage follow each other more rapidly, but are still clearly distinct from the transition to the 7- and 8-cell stage. In the unsynchronized control population both unbudded and budded cells are initially present. Therefore during the first divisions transitions from the single-cell stage to the 2-cell stage occur. Some colonies then reach the 3-, 4- and subsequently higher cell number stages, however there is significant overlap between the different transitions of the unsynchronized control. In addition to validating cell synchronization, these experiments also show yeast viability, since in general all the cells selected by the sorting procedure $(N \approx 30$ for convenient observation in an outlet channel) divide. For the medium conductivity used in the experiments described above, dead cells lack a cross-over in the frequency range considered, ${ }^{20}$ thus selecting the cells with the most negative opacity value also eliminates the dead cells.

\section{Modelling}

In this report, we show that dielectrophoresis involving opposition of forces produced by electrical signals of different frequencies is a suitable tool to synchronize yeast populations. In the following we provide deeper insight into how dielectrophoretic forces act on cultured yeast cells. The physical origin of the dielectrophoretic force is the action of an inhomogeneous field on the dipole moment induced by the very same field on a particle in suspension. The dipole moment, in elementary terms, is given by the product of the accumulated charges of opposite sign times the distance separating them. We therefore expect a contribution from the permittivities of medium and particle, leading to charge accumulation at the interfaces on the one hand, and a purely geometrical contribution reflecting charge separation on the other. This double contribution of composition and geometry is described by the Clausius-Mossotti factor $f_{\mathrm{CM}}$. The ClausiusMossotti factor in turn determines the dielectrophoretic opacity (eqn (2)), and hence ultimately the sorting of the yeast cell cycle stages presented here. In order to understand the theoretical basis of the sorting method presented here, we therefore need to investigate the variation of the Clausius-Mossotti factor throughout the yeast cell cycle.

The detailed expression for the Clausius-Mossotti factor $f_{\mathrm{CM}}$ reflects the contributions of cell composition and cell geometry:

$$
f_{\mathrm{CM}}(\omega)=\frac{\varepsilon_{p}^{*}(\omega)-\varepsilon_{m}^{*}(\omega)}{\varepsilon_{m}^{*}(\omega)+\left[\varepsilon_{p}^{*}(\omega)-\varepsilon_{m}^{*}(\omega)\right] \cdot n_{x}}
$$

The effective complex particle permittivity $\varepsilon_{p}^{*}$ reflects mainly the cell composition - internal structure and material propertieswhereas the form factor $n_{x}$ depends solely on the cell shape. The value of the Clausius-Mossotti factor is also strongly dependent on the conductivity $\sigma_{m}$ of the external suspension medium, since the complex permittivity of the medium $\varepsilon_{m}^{*}$ is a function of both the real permittivity $\varepsilon_{m}$ and the conductivity $\sigma_{m}$ of the medium according to $\varepsilon_{m}^{*}=\varepsilon_{m}+\frac{\sigma_{m}}{j \omega}, j$ being the imaginary unit.

The dependence of the Clausius-Mossotti factor on cell geometry and structure is convenient in our case: during the cell cycle, the yeast cells progressively change geometry, whereas the neck closure is an internal structure change.

Both for the evaluation of effective permittivity $\varepsilon_{p}^{*}$ of the yeast cells and the form factor $n_{x}$, a quantitative description of the evolution of the yeast cell geometry throughout the cell cycle is 
needed. We use a model describing the yeast cells as connected ellipsoids with a rotational symmetry axis, and varying major and minor radii during the cell cycle. The details of this model can be found in Supplementary Information 2. $\$$

Based on this geometrical description and literature values for the electric material constants of cell wall, cell membrane and cytoplasm, we obtain the yeasts' complex permittivity $\varepsilon_{p}^{*}$. The details of the calculation are given in Supplementary Information $3,+$ in particular we evaluate $\varepsilon_{p}^{*}$ from equation 18 of Supplementary Information 3. Fig. 4-A shows the real part of the complex permittivity $\varepsilon_{p}^{*}$, illustrating that the largest differences between the different cell cycle stages are visible at low frequencies, whereas at high frequency, $\varepsilon_{p}^{*}$ depends little on the cell cycle stage.

Although $\varepsilon_{p}^{*}$ does depend on the cell geometry, quantitatively, the main influence of the cell geometry on the Clausius-Mossotti factor arises through the form factor $n_{x}$. The form factor $n_{x}$, also referred to as Lorentz depolarisation factor, can be obtained by calculating the local secondary electric field in relation to the induced dipole moment, ${ }^{23}$ taking into account the exact cell geometry. For the calculation of $n_{x}$, we use our geometrical model of the yeast cells as single or connected ellipsoids for nondividing and dividing yeast cells respectively, given in Supplementary Information 2. For single, closed ellipsoids, formulas for the form factor are available in literature, ${ }^{24}$ for the budding yeast, we provide the calculation in Supplementary Information 4 (namely, eqn (19) in Supplementary Information $4 \ddagger$ ). The resulting form factors throughout yeast growth and division are represented in Fig. 4-B.

For very small cells, we observe a nearly spherical shape, and the form factor corresponds to the theoretical limit of $\frac{1}{3}$ for spherical dielectric bodies. ${ }^{24}$ The cells then start to elongate, which results in a decrease of the form factor. Bud growth induces a drastic further drop of the form factor, due to the globally highly elongated shape of the dividing yeast pair. Eventually, the cells separate and the value of the form factor returns again to $\frac{1}{3}$, the one of single cells.

Taken together, the form factor $n_{x}$ and complex particle permittivities $\varepsilon_{p}^{*}$ allow us to calculate the Clausius-Mossotti factor $f_{\mathrm{CM}}$ for a given medium conductivity and permittivity. Fig. 4-C shows the Clausius-Mossotti factor as a function of signal frequency for different yeast growth stages. Despite the relatively large differences in permittivity at low frequencies (shown in Fig. 4-A), the difference in Clausius-Mossotti factors between the different growth stages is comparatively small in the low frequency range. This is because the Clausius-Mossotti

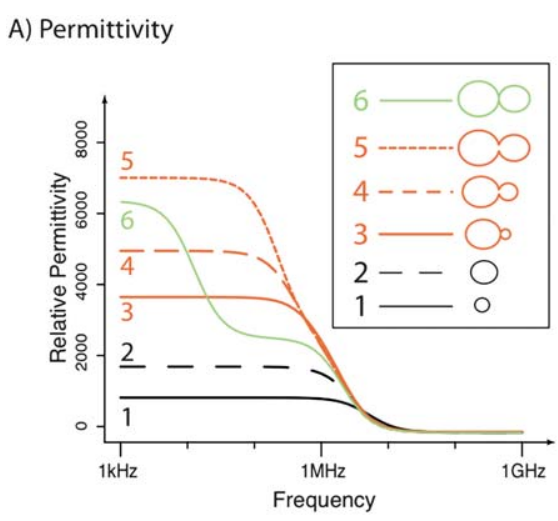

C) Clausius-Mossotti factor

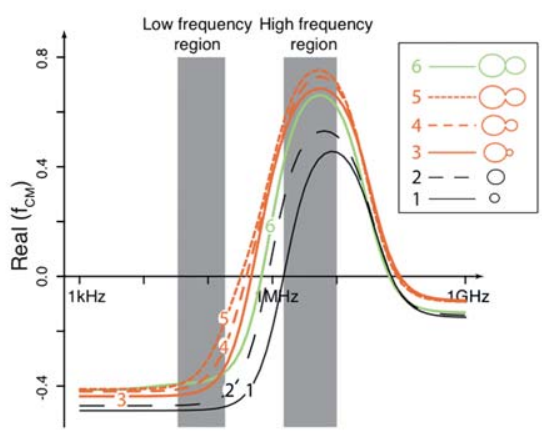

\section{B) Form factor}

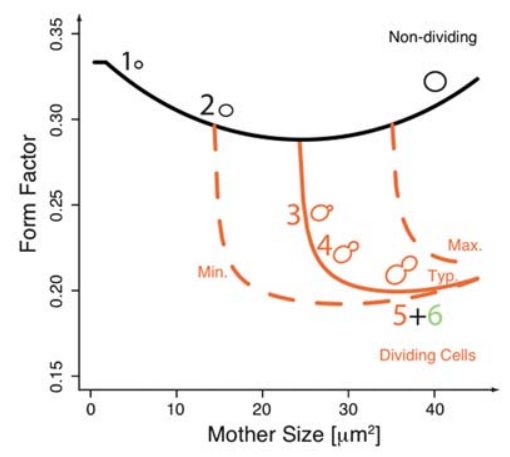

D) Opacity @ $1.5 \mathrm{MHz}$ vs $100 \mathrm{KHz}$

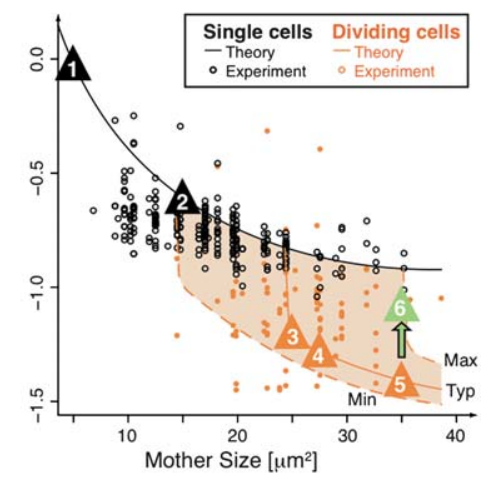

Fig. 4 Dielectric response, form factor and opacities of the yeast cell cycle. (A) Estimation of the complex permittivity of yeast cells over their cell cycle. With cell cycle progression, the permittivity increases (cell geometries 1-5). With diminishing neck diameter in cytokinesis, however, an additional low frequency relaxation appears and the permittivity shifts to lower values (cell geometry 6). (B) Form factor for yeast cells modelled as single or connected ellipsoids. (C) Calculated Claussius-Mossotti factor throughout the yeast cell's division cycle. (D) Experimental and theoretical data for the yeast cell's opacities values throughout their cell cycle for a $100 \mathrm{kHz}$ low frequency and a $1.5 \mathrm{MHz}$ high frequency signal. The opacity reaches a minimum in anaphase (geometry 5), before cytokinesis electrically isolates the cells and restores the opacity to a value close to the one expected for single cells (geometry 6). 
factor depends on the relative magnitude of complex permittivity of both the medium and the suspended cells; at low frequency, the complex permittivity of the medium is comparatively large due to the medium conductivity, so that even large changes in yeast permittivity lead only to small changes for the ClausiusMossotti factor. Nevertheless, there is a low frequency window ranging from about $40 \mathrm{kHz}$ to $500 \mathrm{kHz}$ where significant differences among the division stages exist (lines 3-6 in Fig. 4-C). When going to higher frequency, the Clausius-Mossotti factor becomes positive at about $300 \mathrm{kHz}-1.5 \mathrm{MHz}$, depending on the cell size. At higher frequencies still, the Clausius-Mossotti factor is positive, indicating pDEP for all stages. In a window from about $1.5 \mathrm{MHz}$ to $10 \mathrm{MHz}$, we observe again significant differences between the different dividing stages. With a medium conductivity of $60 \mathrm{mS} \mathrm{m}^{-1}$ used throughout this study, and the requirement for at least one repulsive dielectrophoretic force (nDEP), we therefore identify a low-frequency window from 40 $\mathrm{kHz}$ to $200 \mathrm{kHz}$, and a high frequency window in the range from 1.5 MHz to $10 \mathrm{MHz}$ that should be useful for sorting of the yeast division stages. To enhance the specificity of the method, we generally use both frequency ranges, leading to the sorting strategy employed in this study with a low- and a high-frequency signals combined.

Finally, we provide a direct comparison of calculated and experimentally estimated opacity values in Fig. 4-D, for a 100 $\mathrm{kHz}$ low frequency and a $1.5 \mathrm{MHz}$ high frequency signal. Due to their elongated shape, and therefore the smaller form factors values, the real part of the Clausius-Mossotti factor of the dividing cells is more positive at the high frequency signal (Fig. 4C) and less negative at low frequency (Fig. 4-C). Therefore taking the opacity defined as the high to low frequency ratio of the real part of the Clausius-Mossotti factor leads us to expect more negative opacity values for the dividing cells as compared to the non-dividing cells. The experimental data presented in Fig. 4-D confirms this assumption, and also shows that the change in opacity due to the cell division is larger than opacity changes due to cell size variation. In Fig. 4-D, the different cell division stages are not resolved. Details of the variation of the opacity along the cell division cycle are given in Fig. 2-B.

\section{Conclusions}

Dielectrophoretic separation of yeast cells has been performed in numerous studies, in particular to separate living from dead cells. $^{20,25-28}$ The dielectrophoretic force is highly sensitive to the cell size, since the force is to a first approximation proportional to the volume. Indeed, for cells with little shape variation, the size dependence of the dielectrophoretic force can directly be used to sort different division stages because of the steady increase in cell size before division. ${ }^{17}$ For yeast cells, the situation is more complicated because of the budding process, which leads to a change in shape during growth. We have recently developed equilibrium-based approaches to dielectrophoretic separation, where the direct volume dependency cancels out. ${ }^{19}$ Here we show that it is possible to characterize and continuously sort different yeast cell division stages using this equilibrium-based dielectrophoresis approach. We characterize the different cell cycle stages in terms of the opacity as defined by the ratio of dielectrophoretic forces at two different frequencies. Modelling finally illustrates the interplay between internal electric properties and the cell shape: bud growth leads to a more elongated global cell shape, and a higher dipole moment in particular at high frequencies, whereas cell abscission physically and thus electrically isolates mother and daughter cell, returning the electrical properties close to what would be observed for single cells.

Cell sorting is performed here in a continuous flow system designed to allow direct experimental estimation of the opacity from the lateral positions of the cells after the sorting element. The sorting rates achievable are on the order of 100 cells s $^{-1}$. To analyze larger cell numbers, it may be more advantageous to use open structures, ${ }^{29}$ several sorting elements in parallel, or $3 \mathrm{D}$ electrode structures. ${ }^{30,31}$ The theoretical framework allowing to choose suitable electrical signals remains valid for sorting geometries adapted to different purposes.

Finally, the method is label- and contact-free and does not employ drugs interfering with the cell metabolism. Like elutriation, it selects cells from a given population rather than arresting the population in a particular stage. As it selects cells in a later stage than elutriation, it should open up the way for combined schemes such as selection for fast or slow dividers. In view of extending our understanding of the gene networks that produce and regulate regular cyclic cellular behaviour, the ease of integration of electrical actuation methods in microfluidic schemes should allow to devise complex selection procedures for obtaining cells with a particularly stable, alternating or even irregular, bet hedging ${ }^{32}$ type of cycle.

\section{Methods}

\section{Microfluidic device design and operation}

The microfluidic device consists of a network of photopatterned channels in SU8 with Ti-Pt electrodes previously structured by a lift-off process. The chip is reversibly sealed by a molded PDMS block containing embedded liquid reservoirs and the full device is mounted into a custom-made fluidic and electrical interface. ${ }^{33,34}$ More details can be found in..$^{20}$

\section{Yeast culture and sample preparation}

The yeast cells are cultured at $20^{\circ} \mathrm{C}$ on YPD agar plates $1000 \mathrm{ml}$ of deionised water, $10 \mathrm{~g}$ of BactoYeast Extract, $20 \mathrm{~g}$ of BactoPeptone, $20 \mathrm{~g}$ of dextrose and $20 \mathrm{~g}$ of agar). For the experiments, cells in logarithmic growth phase are transferred to YPD liquid media which is adjusted with deionised water to a conductivity of $60 \mathrm{mS} \mathrm{m}{ }^{-1}$, after dissociation of larger clusters by vortexing and centrifugation, followed by $30 \mathrm{~min}$ incubation prior to on-chip sorting.

\section{GFP tubulin yeast}

The GFP-Tub1 fusion under the control of the endogenous promoter was constructed as previously described. ${ }^{35,36}$ The resultant GFP-Tub1 fusion was integrated at the URA3 locus after Stu I digestion. 


\section{Image acquisition and analysis}

The microfluidic device was mounted on to an XYZ translation stage in an inverted microscope (Leica DMIL, Leica Microsystems, Wetzlar, GmbH, Germany). The microscope system is equipped with several different magnification objectives and a computer-controlled camera (uEye) is mounted in the microscope for image/video recording.

For the GFP-tubulin yeast experiments the cell sorting microfluidic device was made on a thin-glass wafer $(170 \mu \mathrm{m}$ thick) and placed on a motorized inverted microscope Olympus fluorescence (IX81S1F-ZDC) equipped with a stage incubator. Images were taken with $100 \times$ oil inversion objective and acquired with a CCD camera (Hamamatsu ORCA/ER) using the fluorescence GFP filter.

\section{Acknowledgements}

We wish to thank Dr Arnaud Bertsch and Harald Van Lintel for proofreading the manuscript. This work was in part funded by the European Community CellPROM project under the 6th Frame-work Program, contract No. NMP4-CT-2004-500039.

\section{References}

1 L. Hartwell, Saccharomyces cerevisiae cell cycle 1974, Bacteriol. Rev., 1974, 38, 164-198.

2 I. Herskowitz, Life cycle of the budding yeast saccharomyces cerevisiae, Microbiol. Mol. Biol. Rev., 1988, 52, 536-553.

3 S. Martin, Geometric control of the cell cycle, Cell Cycle, 2009, 8, 3643-3647.

$4 \mathrm{M}$. Snyder and J. Gallagher, Systems biology from a yeast omics perspective, FEBS Lett., 2009, 583, 3895-3899.

5 B. Futcher, Cell cycle synchronization, Methods Cell Sci., 1999, 21, 79-86.

6 P. T. Spellman, et al. Comprehensive identification of cell cycleregulated genes of the yeast saccharomyces cerevisiae by microarray hybridization, Mol. Biol. Cell, 1998, 9, 3273-3297.

7 S. A. Moore, Comparison of dose-response curves for alpha factorinduced cell division arrest, agglutination, and projection formation of yeast cells. implication for the mechanism of alpha factor action, J. Biol. Chem., 1983, 258, 13849-13856.

8 I. Fitch, et al. Characterization of four b-type cyclin genes of the budding yeast saccharomyces cerevisiae, Mol. Biol. Cell, 1992, 3, $805-818$.

9 G. M. Walker, Synchronization of yeast cell populations, Methods Cell Sci. 1999, 21, 87-93.

$10 \mathrm{~K}$. Shedden and S. Cooper, Analysis of cell-cycle gene expression in saccharomyces cerevisiae using microarrays and multiple synchronization methods, Nucleic Acids Res., 2002, 30, 29202929.

$11 \mathrm{~A}$. Goranov, et al. The rate of cell growth is governed by cell cycle stage, Genes Dev., 2009, 23, 1408-1422.

$12 \mathrm{~K}$. Asami, E. Gheorghiu and T. Yonezawa, Real-time monitoring of yeast cell division by dielectric spectroscopy, Biophys. J., 1999, 76, 3345-3348.

13 H. A. Pohl. Dielectrophoresis, Cambridge University Press, 1978.

$14 \mathrm{H}$. Morgan and N. G. Green. AC Electrokinetics: Colloids and Nanoparticles, Research Studies Press, 2002.
15 R. Pethig, Dielectric properties of biological materials: Biophysical and medical applications, IEEE Trans. Electr. Insul., 1984, EI-19, 453-474.

$16 \mathrm{H}$. Schwan, Electrical properties of tissue and cell suspensions, $A d v$. Biol. Med. Phys., 1957, 5, 147-209.

17 U. Kim, et al. Selection of mammalian cells based on their cell-cycle phase using dielectrophoresis, Proc. Natl. Acad. Sci. U. S. A., 2007, 104, 20708-20712.

18 N. Demierre, T. Braschler, R. Muller and P. Renaud, Focusing and continuous separation of cells in a microfluidic device using lateral dielectrophoresis, Sens. Actuators, B, 2008, 132, 388-396.

19 A. Valero, T. Braschler, N. Demierre and P. Renaud, A miniaturized continuous dielectrophoretic cell sorter and its applications, Biomicrofluidics, 2010, 4, 022807-9.

$20 \mathrm{~T}$. Braschler, et al. Continuous separation of cells by balanced dielectrophoretic forces at multiple frequencies, Lab Chip, 2008, 8, 280-286.

21 S. Shaw, P. Maddox, R. Skibbens, E. Yeh, K. Salmon and E. D. Bloom, Nuclear and spindle dynamics in budding yeast, Mol. Biol. Cell, 1998, 9, 1627-1631.

$22 \mathrm{M}$. Winey and E. O'Toole, The spindle cycle in budding yeast, Nat. Cell Biol., 2001, 3, E23-27.

23 J. Maxwell Garnett, Colours in metal glasses and metal films, Trans. of the Royal Society CCIII, 1904, 384 420.

24 A. Sihvola. Electromagnetic mixing formulas and applications, The Institution of Electrical Engineers, 1999.

25 G. H. Markx, M. S. Talary and R. Pethig, Separation of viable and non-viable yeast using dielectrophoresis, J. Biotechnol., 1994, 32, 29-37.

26 G. H. Markx and R. Pethig, Dielectrophoretic separation of cells: Continuous separation, Biotechnol. Bioeng., 1995, 45, 337343.

27 Y. Li, C. Dalton, H. Crabtree, G. Nilsson and K. Kaler, Continuous dielectrophoretic cell separation microfluidic device, Lab Chip, 2007, 7, 239-248.

28 G. Mernier, N. Piacentini, R. Tornay, N. Buffi and P. Renaud, Labelfree sorting and counting of yeast cells for viability studies, Procedia Chem., 2009, 1, 385-388. Proceedings of the Eurosensors XXIII conference.

29 C. Jen and T. Chen, Trapping of cells by insulator-based dielectrophoresis using open-top microstructures, Microsyst. Technol., 2009, 15, 1141-1148.

30 L. Wang, L. Flanagan, N. Jeon, E. Monuki and A. Lee, Dielectrophoresis switching with vertical sidewall electrodes for microfluidic flow cytometry, Lab Chip, 2007, 7, 1114-1120.

31 L. Wang, et al. Dual frequency dielectrophoresis with interdigitated sidewall electrodes for microfluidic flow-through separation of beads and cells, Electrophoresis, 2009, 30, 782-791.

32 H. Beaumont, J. Gallie, C. Kost, G. Ferguson and P. B. Rainey, Experimental evolution of bet hedging, Nature, 2009, 462, 9093.

33 T. Braschler, Controlled Entrapment of Cells in Hydrogels on Chip and Cell Sorting by Dielectrophoresis using Liquid Electrodes, Ph.D. thesis, Ecole Polytechnique Federal de Lausanne, 2009.

34 N. Demierre, Continuous-flow Separation of cell in a lab-on-a-chip using "Liquid electrodes" and Multiple Frequency Dielectrophoresis, $\mathrm{Ph} . \mathrm{D}$. thesis, Ecole Polytechnique Federal de Lausanne, 2009.

35 A. Straight, A. Belmont, C. Robinett and A. Murray, Gfp tagging of budding yeast chromosomes reveals that protein-protein interactions can mediate sister chromatid cohesion, Curr. Biol., 1996, 6, 15991608.

36 A. Straight, W. Marshall, J. Sedat and A. Murray, Mitosis in living budding yeast: anaphase a but no metaphase plate, Science, 1997, 277, 574-578. 